\title{
Cuidado cultural familiar al paciente diabético que consume alcohol y/o tabaco
}

\section{Cultural family care to diabetic patients who consumed alcohol and/or tobacco}

\section{Cuidado cultural familiar ao paciente diabético que consome álcool e/ou tabaco}

\author{
Leticia Casique Casique ${ }^{1}$, Teresita de Jesús Muñoz Torres², Blanca Yuliana Castro García ${ }^{3}$, \\ Zuleima Centeno González ${ }^{3}$; Rodrigo López Franco ${ }^{3}$
}

${ }^{1}$ Doctora en Ciencias de Enfermería. Docente e Investigador de la Facultad de Enfermería y Obstetricia de Celaya, Universidad de Guanajuato. Av. Ing. Javier Barros Sierra No. 201 Esquina Av. Baja California. Ejido de Santa María del Refugio, Celaya Gto. C.P.38110.E-mail:E-mail leticiacc_2004@yahoo.com.mx.

${ }^{2}$ Maestra en Ciencias de Enfermería. Profesor Hora-Clase de la Unidad Académica Multidisciplinaria Zona Media de la Universidad Autonomía de San Luis Potosí.

${ }^{3}$ Estudiante de la Licenciatura en Enfermería y Obstetricia, Universidad de Guanajuato Campus Celaya-Salvatierra. Cómo citar este artículo en edición digital: Casique Casique, L., Muñoz Torres, T.J., Castro García, B.Y., Centeno González, Z., \& López Franco, R. (2017). Cuidado cultural familiar al paciente diabético que consume alcohol y/o tabaco. Cultura de los Cuidados (Edición digital), 21(49). Recuperado de http://dx.doi.org/10.14198/cuid.2017.49.14

Correspondencia: Universidad de Guanajuato. Av. Ing. Javier Barros Sierra No. 201 Esquina Av. Baja California. Ejido de Santa María del Refugio, Celaya Gto. C.P. 38110.

Correo electrónico: leticiacc_2004@yahoo.com.mx Recibido: 08/02/2017; Aceptado: 24/05/2017

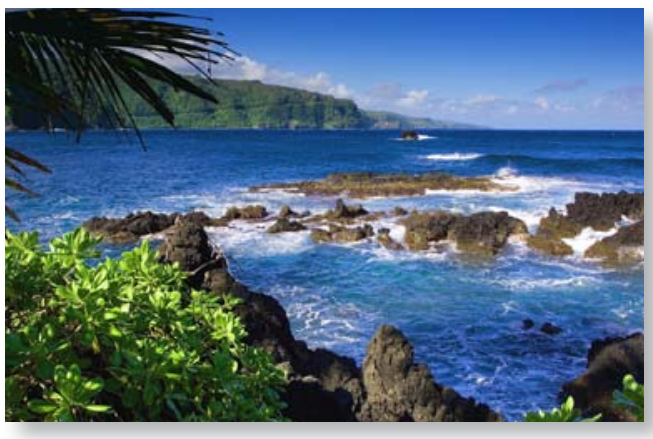

\section{ABSTRACT}

Objective: Understand the cultural care provided by family to the diabetic patient consuming the alcohol and/or tobacco.

Methodology: Analytical and qualitative study, we conducted analysis of speech, with a final sample of 9 subjects with Diabetes; the data collected from the survey "Cultural family care to diabetic patients who consumed alcohol and/or tobacco" to apply through a focus group. The personal data were analysed using the statistical package SPSS v.17.0 and the qualitative data were analyzed based on the Grounded of Data Theory.

Results: The phenomenon found is an optimal and comprehensive family care, which manifests itself by na excellent behavioral expression and verbal, coupled with feelings of gratitude by the individual.

Conclusion: The family care provided to diabetic patients an emotional, social, physical and spiritual level is essential to strengthen the provision in adherence to treatment, and to face the mourning by the loss of a lifestyle.

Keywords: Cultural care, diabetes type 2, nursing.

\section{RESUMEN}

Objetivo: Comprender el cuidado cultural que la familia proporciona al paciente diabético que consume alcohol y/o tabaco. 
Metodología: Estudio cualitativo y analítico, se realizó análisis de discurso, con una muestra final de 9 sujetos con Diabetes; los datos se recolectaron a partir de la encuesta "Cuidado cultural familiar al paciente diabético que consume alcohol y/o tabaco" aplicándose a través de un grupo focal. Los datos personales se analizaron en el paquete estadístico SPSS v.17.0 y en los datos cualitativos se analizaron con base en la Teoría Fundamentada de los Datos.

Resultados: El fenómeno encontrado es cuidado familiar óptimo e integral, que se manifiesta por una excelente expresión conductual y verbal, aunado a sentimientos de gratitud por parte del individuo.

Conclusión: El cuidado que los familiares proporcionan al paciente diabético a nivel emocional, social, físico y espiritual es fundamental para fortalecer la disposición en el apego al tratamiento, y para afrontar el duelo por la pérdida de un estilo de vida.

Palabras clave: Cuidado cultural, diabetes tipo 2, enfermería.

\section{RESUMO}

Objetivo: Compreender o cuidado cultural que a família proporciona ao paciente diabético que consome álcool e/ou tabaco.

Metodologia: Estudo qualitativo, analítico, que utilizou análise de discurso, com 9 participantes com diabete. Os dados foram coletados com base no instrumento "Cuidado cultural familiar al paciente diabético que consume alcohol y/o tabaco" por meio de grupo focal. Os dados pessoais foram analisados com o pacote estatístico SPSS v.17.0 e os dados qualitativos foram analisados com base na Teoria Fundamentada em Dados.

Resultados: $\mathrm{O}$ fenômeno encontrado é o cuidado familiar ótimo e integral, que se mani-

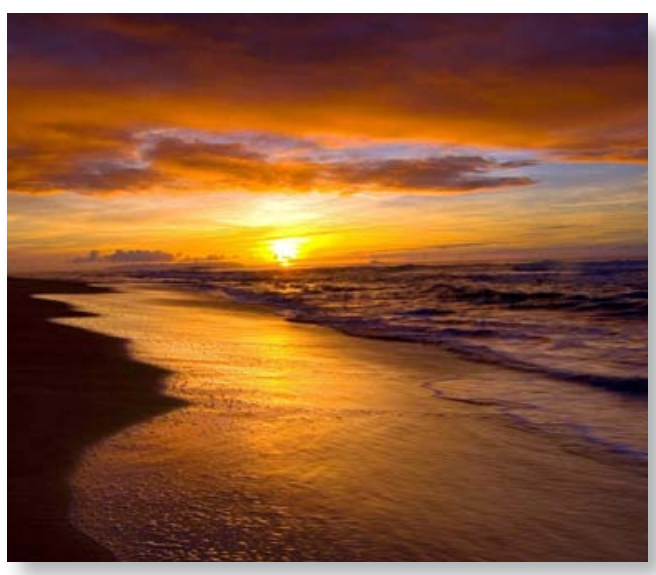

festa por uma excelente expressão de conduta e verbal, somando a sentimentos de gratidão por parte do indivíduo.

Conclusão: $O$ cuidado que os familiares proporcionam ao paciente diabético nos níveis emocional, social, físico e espiritual é fundamental para fortalecer a disposição de apego ao tratamento e para afrontar o duelo pela perda de um estilo de vida.

Palavras chave: Cuidado cultural, diabetes tipo 2, enfermagem.

\section{INTRODUCCIÓN}

La Diabetes es una de las enfermedades crónico-degenerativas de mayor preponderancia, un reflejo de esta problemática son las 346 millones de personas en el mundo que la padecen, y en donde más del $80 \%$ de las muertes por diabetes se registran en países con ingresos bajos y medios, situación que podría multiplicarse entre los años 2005 y 2030 (ONU, 2012). En México, existen 4.2 millones de personas con Diabetes según los datos proporcionados por el Instituto Mexicano del Seguro Social en el 2009 (IMSS, 2009), esto como resultado del envejecimiento de la población y del incremento de los riesgos asociados a la industrialización y urbanización del estilo de vida, 
denominándose a estos fenómenos como problemas emergentes, puesto que afectan a todos los sectores de la sociedad.

Dicho problema se agrava cuando las personas con Diabetes consumen alcohol y tabaco, ya que según datos de la Organización Mundial de la Salud, durante el período 20022011 se incrementó la dependencia al alcohol, de 3.5 a $6.2 \%$ en los hombres mientras que en la población femenina los números se triplicaron de un 0.6 a 2\%; en relación al tabaco, las cifras se sitúan entre los 29.8 y 32.2 millones de adultos mayores de 20 años (Universia México, 2014; Guerrero, 2013),por lo que se establece que el $18.5 \%$ de la población mexicana entre 12 y 65 años de edad son fumadores (Secretaria de Salud, 2001). Por su parte, en Guanajuato existen 458 mil casos de Diabetes, mientras que la Unidad Médica de Atención Primaria a la Salud (UMAPS) Franco-Tavera, ubicada en el municipio de Juventino Rosas Guanajuato, reportó un total de 62 casos de Diabetes en el mes de Abril del 2012.

La práctica del consumo de alcohol, es en cierta forma resultado de la cultura que rodea al individuo, y que se refleja en el consumo nocivo de bebidas alcohólicas, situación que causa 2.5 millones de muertes cada año. El consumo de alcohol ocupa el 3er lugar entre los factores de riesgo de la carga mundial de morbilidad, y es el primer factor en la Américas; siendo este una variable causal de problemas graves de índole social y de desarrollo, entre los que se encuentran la violencia, el descuido, maltrato de menores y el ausentismo laboral (ONU, 2012). El consumo de tabaco también representa un problema de salud pública en México, ya que 14 millones de personas realizan esta práctica (Guerrero, 2013), cifra en sí alarmante por el gran número de personas diabéticas que forman parte de estos datos.
La preocupación sobre estos cambios conductuales en las personas diabéticas radica en que cuanto mayor sea el consumo de alcohol y/o tabaco mayor será el riesgo de sufrir alguna de las complicaciones inherentes a la enfermedad, como es infarto al miocardio, retinopatía, hipoglucemia, entre otras; así como aquellas que son provocadas por el consumo de alcohol y tabaco como la cirrosis hepática, Síndrome de Korsakoff, Enfermedad Pulmonar Obstructiva Crónica, entre otras.

Ante esta problemática, la sociedad ha desarrollado cuidados empíricos, según la cultura en que se desenvuelve la persona con diabetes y su familia, para ayudar a mejorar la salud del familiar enfermo. Partiendo de lo antes descrito, es que el presente trabajo tiene como objetivo proponer una red conceptual sobre el cuidado cultural que la familia proporciona al paciente diabético que consume alcohol y/o tabaco, del Grupo de Ayuda Mutua (GAM), del UMAPS Franco-Tavera, del Municipio de Juventino Rosas, Guanajuato.

\section{METODOLOGÍA}

Estudio cualitativo y analítico, se realizó analisis de discurso y se aplicó la Teoría Fundamentada en los Datos, realizado del 2 de Abril al 30 de Noviembre de 2012 con una muestra final de 9 individuos diagnosticados con Diabetes y pertenecientes al GAM de la UMAPS Franco-Tavera ubicado en la localidad de Juventino Rosas, Guanajuato; la selección de los sujetos fue mediante un muestreo aleatorio y respetando los criterios de inclusión (personas diabéticas que consuman alcohol y/o tabaco, entre 40 y 80 años de edad y que aceptaran participar en la investigación). Para la recolección de datos se utilizó la encuesta "Cuidado cultural familiar al paciente diabético que consume alcohol y/o tabaco" conformada por tres 


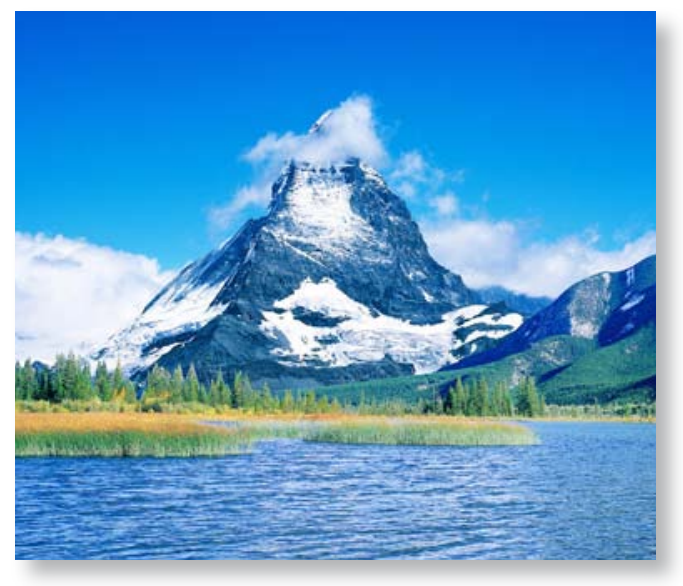

apartados: 1) Datos personales como son la edad, género, estado civil, ocupación, religión y escolaridad; 2) Datos del cuidador haciendo un énfasis particular en la edad, género, estado civil, ocupación, escolaridad y parentesco; y 3) Cuidado cultural en donde el paciente debía explicar cómo lo cuida su familiar y/o persona con vínculo emocional en el aspecto físico, psicológico, social y espiritual, de donde adquirió el familiar esos conocimientos y qué piensa del cuidado que le proporciona su familiar. El procedimiento del estudio comprendió la aplicación del instrumento mediante la dinámica de grupo focal dirigida por un coordinador y apoyada por cuatro observadores. Una vez recolectados los datos se continuó con el análisis mediante la utilización del programa SPSS en su versión 17 para los datos descriptivos, a su vez se aplicó la Teoría Fundamentada de los Datos para el análisis correspondiente de los datos cualitativos.

\section{RESULTADOS}

En el estudio se encontró que el $88.9 \%$ de los individuos diabéticos son masculinos y su estado civil casado, la media de edad se situó en los 59 años con una $\mathrm{DE}+10.49$, presentando un promedio de 8 años de padecer diabetes, el 55.6\% refirió ser campesino, 22.2\% son anal- fabetas y el resto de la población cuenta con educación primaria, el 100\% manifestó profesar la religión católica y tener un ingreso económico de \$2,200.00 pesos mensuales. En relación al consumo de alcohol este presentó una media de 30 años; por su parte, el consumo de tabaco se presentó en el $44.4 \%$ de la población, siendo predominante en el género masculino.

Respecto al cuidador, el $100 \%$ son del género femenino, con una media de 57 años de edad y una DE +17.2 , el $100 \%$ se dedica a las labores del hogar, el $77.8 \%$ son esposas, 55.6\% son analfabeta y el $44.4 \%$ de dichos cuidadores presenta una enfermedad crónico-degenerativa (Diabetes y/o Hipertensión Arterial).

Se aplicó la correlación de Pearson entre la variable edad con el ingreso económico arrojando una correlación negativa moderada de -.609 con una significancia de .041 lo que indica que a mayor edad menor ingreso económico, esto es entendible ya que la ocupación del diabético es campesino, empleado y/o ama de casa, generando estos empleos una cantidad de recursos económicos más limitados, influyendo estos a su vez, sobre la calidad del cuidado $\mathrm{y}$ autocuidado que se brinda.

En relación con los datos cualitativos en base a la Teoría Fundamentada de los Datos se elaboró un mapa conceptual donde se describe el origen de las categorías a partir de los aspectos físico, espiritual, social y psicológico para posteriormente crear los códigos abiertos que unidos con el código vivo forman el código axial, que permiten establecer el juicio y definir una categoría para cada conjunto de los elementos del código axial, mismos que pueden identificarse en la figura No. 1 y los cuales están relacionados con la alimentación, higiene, actividad física, estabilidad emocional, entre otras. Para profundizar en el análisis se elaboró un mapa conceptual (Fig. No. 2) a partir 
del cual se definen las categorías conformadas por los diversos códigos axiales, que han sido establecidos en la figura 1.A partir del análisis de los diversos códigos, de los juicios y cate- gorías se concluyó con la formulación de una red conceptual en la cual se describe el cuidado familiar al paciente diabético que consume alcohol y/o tabaco (Fig. No. 3).

Figura 1. ORIGEN DE LAS CATEGORÍAS

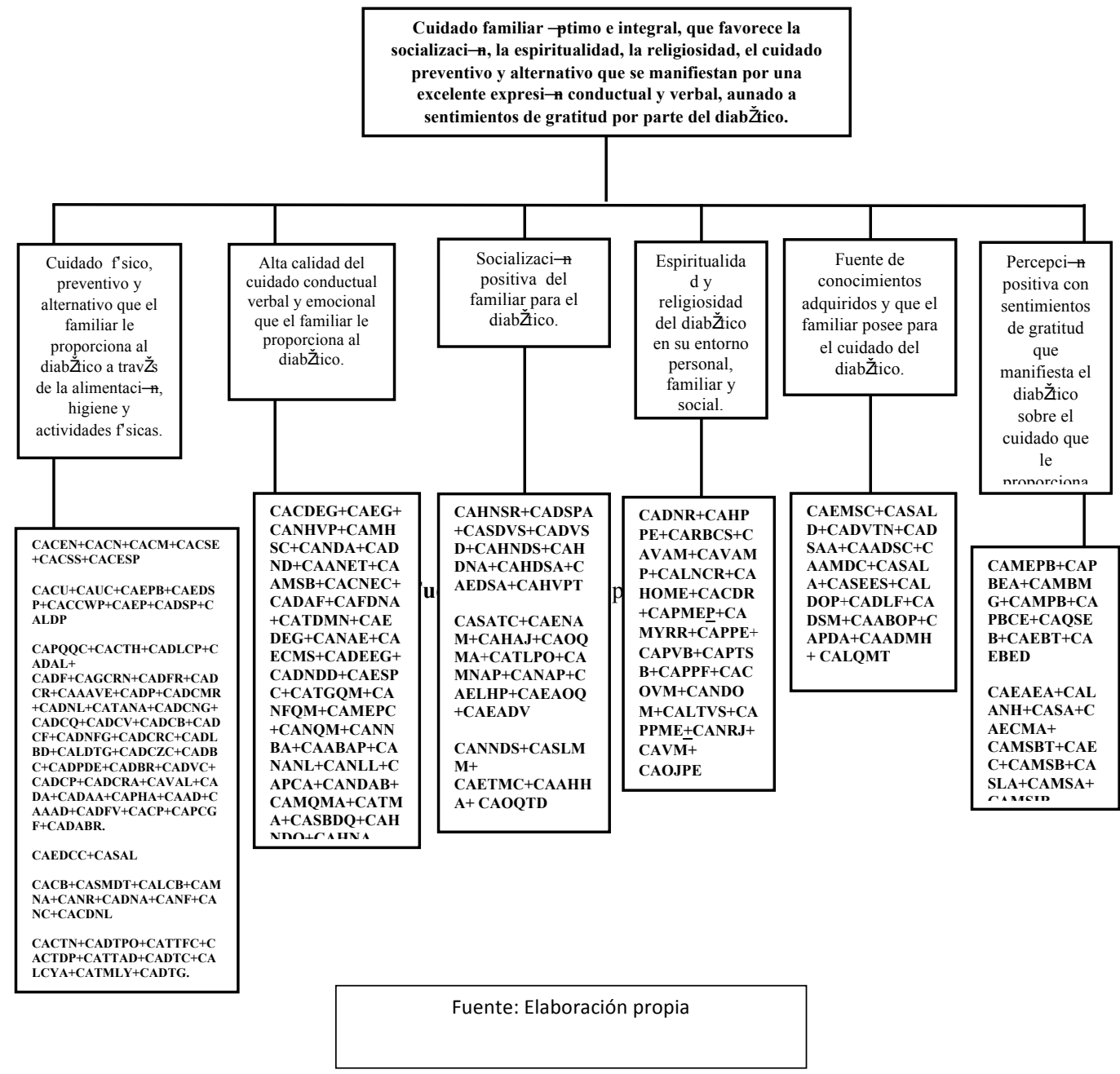


Figura 2 DEFINIIÓN DE LAS CATEGORÍAS

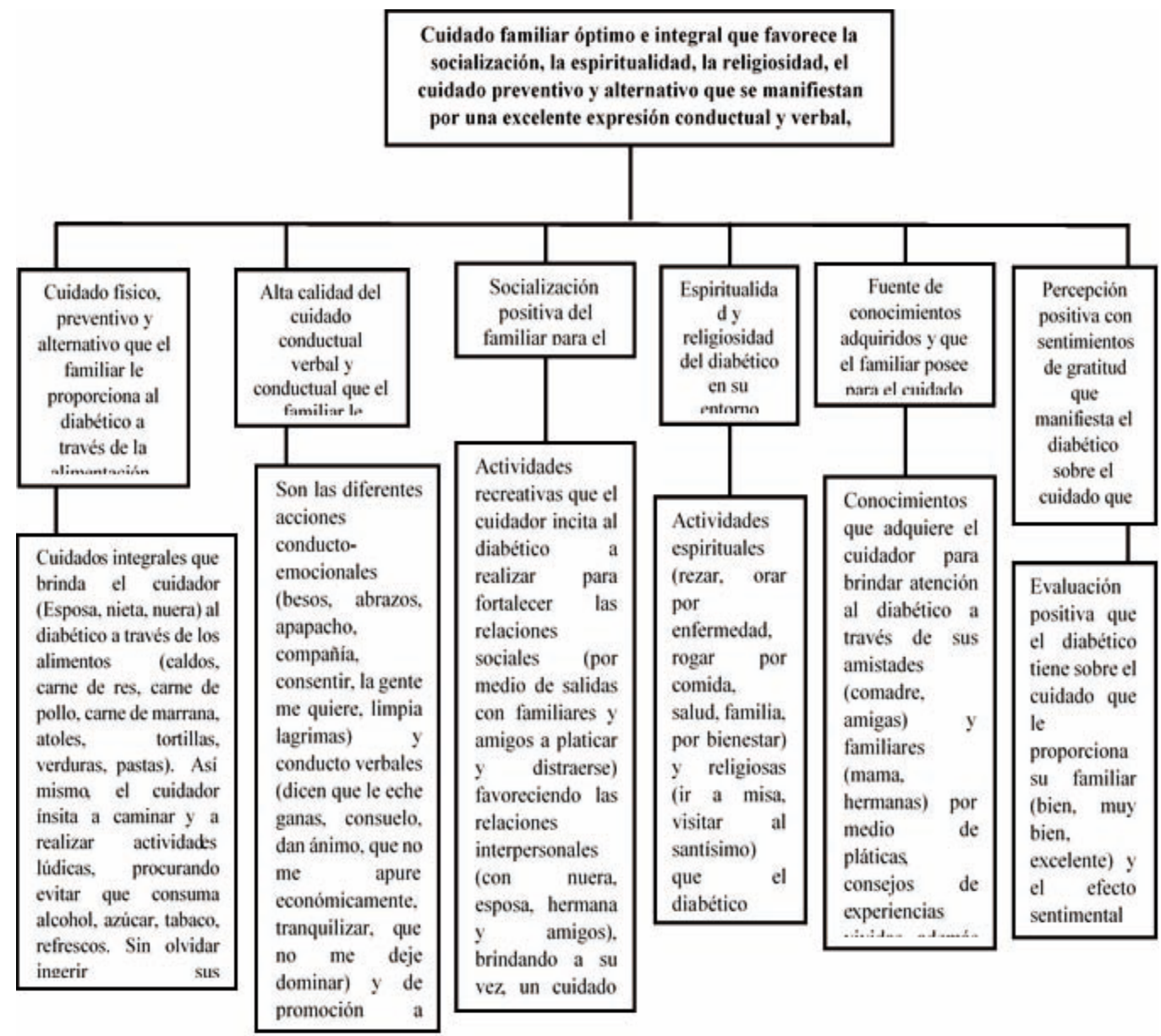

Fuente: Elaboración propia 
Figura 3. RED CONCEPTUAL DEL CUIDADO FAMILIAR AL PACIENTE DIABÉTICO
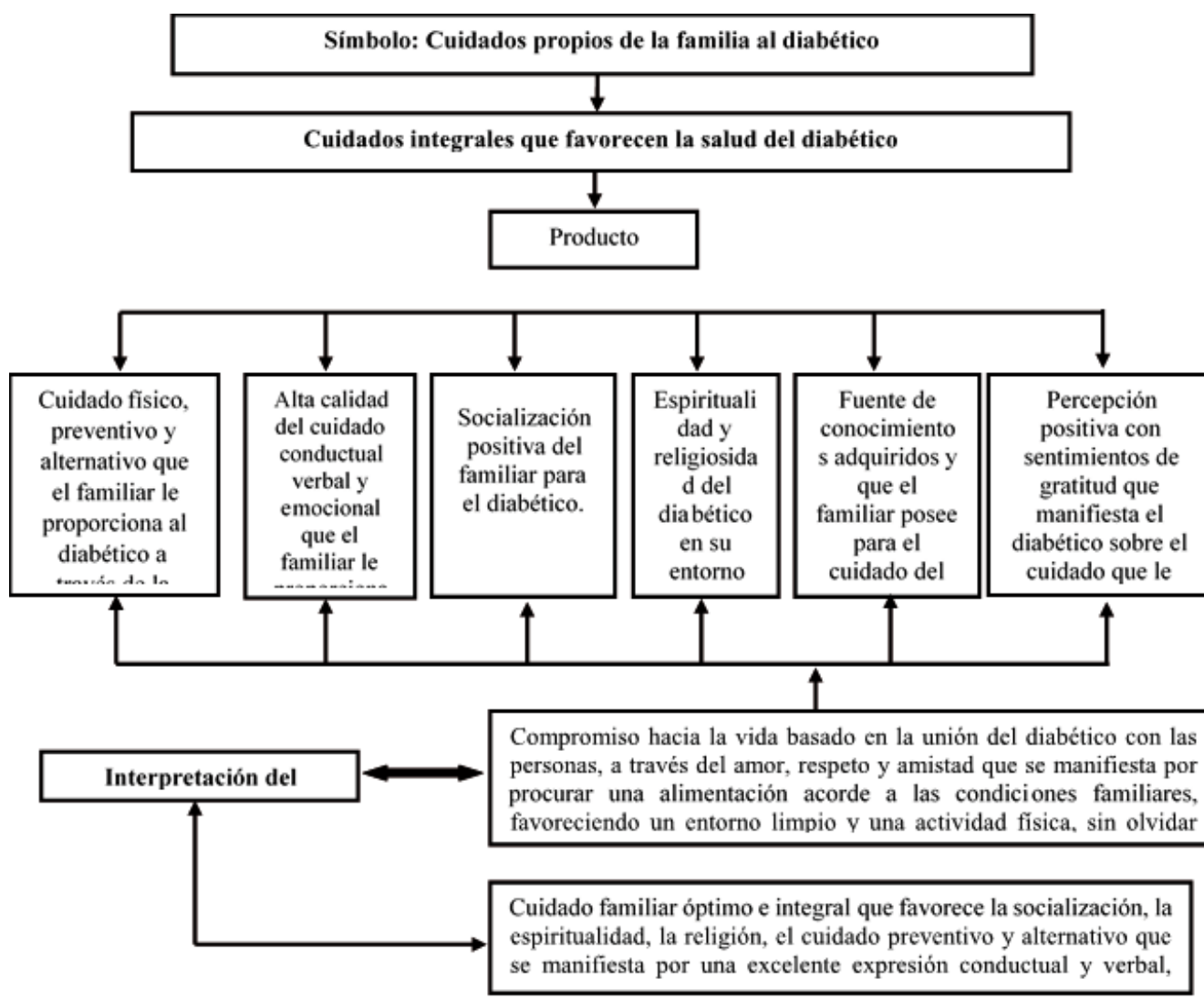

Fuente: Elaboración propia

\section{DISCUSIÓN}

En relación a la edad, la media del presente estudio fue de 59 años ( $\mathrm{DE}=10.493)$; éstos resultados concuerdan con el estudio realizado por Zavala, et. al., donde se presentó un rango de edad entre los 50 a 64 años, con una media de $57.36(\mathrm{DE}=11.40)$ y Jacoby en 2003 , en el cual estableció que personas de 60 años de edad o mayores tienen la prevalencia más alta de enfermedades no transmisibles entre las cuales se encuentra la Diabetes tipo 2 (Zavala, 2006; Jacoby, 2003).

Con respecto al género, se encontró que el 88.9\% de los participantes con Diabetes pertenece al género masculino y el $11.1 \%$ al género femenino. Datos que discrepan con otros estudios de investigación realizados por Solís, et. al. y Zavala, et. al., los cuales revelan que es el género femenino quien predomina con un $52.8 \%$ y un $54.7 \%$ respectivamente (Solís, 
2009; Zavala, 2006); por otro lado, el programa de acción especifico de Diabetes Mellitus 2007-2012 refiere que en la Encuesta Nacional de Salud (ENSA) 2000 se reportó una prevalencia mayor en mujeres $(7.8 \%)$ con respecto a los hombres (7.2\%) (Secretaria de Salud, 2008), lo que conlleva a pensar que se ha presentado un incremento en el número de mujeres que desarrollan Diabetes, posiblemente por un estilo de vida inapropiado (consumo de alcohol, tabaco, dieta inadecuada, ayuno prolongado, grado de escolaridad mínimo que limita la adquisición de conocimientos, etc.).

El $88.9 \%$ de los sujetos de estudio y su cuidador refieren estar casados, dato significativo en esta investigación, ya que Fernández estableció en el 2007, que la Diabetes tiene un gran impacto dentro del núcleo familiar, debido a que la aparición de una enfermedad aguda, crónica o terminal en alguno de los miembros de la familia puede representar un serio problema tanto en su funcionamiento como en su composición, produciendo una crisis y desorganización, que termina impactando en cada miembro del núcleo en los aspectos biopsicosociales y económicos (Fernández, 2007).

Respecto a la religión y espiritualidad, Rondon en el 2011 afirma que éstas son una importante estrategia de afrontamiento para incrementar las conductas saludables y de ajuste a la enfermedad; dicha información coincide con la proporcionada por los sujetos de ésta investigación, los cuales llevan a cabo actividades espirituales (rezar, orar por su enfermedad, su salud y bienestar) y religiosas (ir a misa y visitar al santísimo) que el diabético practica con su familiar para contribuir en su bienestar integral (Rondón, 2011), por lo tanto se puede establecer que para el paciente el contexto espiritual es un factor protector para adherirse de una forma más eficiente a su tra- tamiento farmacológico, dietético y en lo que respecta a la actividad física.

Otro punto importante dentro de este estudio es el nivel de escolaridad de los cuidadores, ya que la educación influye de manera importante en el afrontamiento efectivo de la enfermedad, y más aún cuando el cuidador requiere de esa habilidad de cuidado la cual incluye la dimensión cognoscitiva, según las palabras de Díaz y cols (Díaz, 2009).

Estas relaciones familiares que pueden establecerse entre el género femenino y masculino, de acuerdo al estudio de Salcedo (2008), son generadoras para recibir y dar cariño, afecto y ayuda al diabético en el aspecto psicológico (Salcedo, 2008), con dichos resultados se puede concluir, que el apoyo emocional y el cuidado que los familiares proporcionan al diabético son un elemento fundamental que fortalece la disposición para el apego al tratamiento.

Por otro lado, Zavala en su estudio menciona que los participantes tenían de 1 a 30 años de padecer Diabetes, más del $50 \%$ ya presentaban algún tipo de complicación y en su mayoría con 10 años de evolución (Zavala, 2006), dato que coincide con los resultados obtenidos en el presente estudio, en el cual se identificó una media de 8 años de evolución de la enfermedad; ante esta situación, el personal de salud debe enfatizar en la capacitación sobre el conocimiento de dicho padecimiento, y de esta manera evitar posibles complicaciones con un manejo oportuno y adecuado, y así preservar el grado más alto de salud de las personas con diabetes.

En 2011, Galván Zarate menciona que el consumo de alcohol se ha presentado desde décadas, empero, en la actualidad cobra mayor relevancia el que las personas que consumen alcohol padezcan Diabetes, ya que 
puede disminuir su estado de salud y calidad de vida, afectándose ellos mismos y a su(s) cuidador(es), así como al sistema de salud ya que los gastos por atender las complicaciones son mayores si los comparamos con los utilizados para promover un cuidado integral al paciente diabético. Gómez Aguilar refiere que "es muy cierto que para las personas con Diabetes es más fácil permanecer con viejos hábitos arraigados y asociados a factores sociales y culturales, que adquirir y mantener nuevos conocimientos y actividades que les permitirán mejorar su estado de salud; aun cuando se ha demostrado que el estilo de vida saludable tiene un relación inversamente proporcional al desarrollo de esta enfermedad" (Galván, 2011; Gómez, 2010).

En relación con el consumo de tabaco, el estudio de Gómez Aguilar reportó que el $80.3 \%$ de los sujetos no tienen el hábito de fumar, pero también hace referencia que del total de la muestra, el 75.7\% son mujeres (Gómez, 2010); confrontando los resultados se llegó a la conclusión de que efectivamente las mujeres tienen hábitos menos perjudiciales para la salud por cuestiones sociales y culturales inherentes al medio donde se desarrollan.

Como parte del estudio, el diabético refirió que su cuidador le proporciona productos naturales para complementar el tratamiento farmacológico, entre las que destacan: té de Warequi, nopal, hierbas amargosas y tés (manzanilla, hierbabuena, limón, planta de orquídea, entre otros); cabe mencionar que el cuidador adquirió este conocimiento por parte de sus amigas, mamá, hermanas y medios de comunicación, lo cual coincide con lo establecido por Polit y Hungler, al referir que las fuentes para la adquisición de conocimientos son la tradición, autoridad, experiencia y el ensayo-error (Polit, 2000). En el estudio los participantes descri- bieron que el cuidador motiva a la práctica de actividades físicas como el caminar y participar en actividades lúdicas, lo cual coincide con lo establecido por Gómez, quien menciona que para conseguir un adecuado equilibrio del control glucémico en el tratamiento de la diabetes se han considerado como pilares a la medicación y los estilos de vida (Gómez, 2010), en este último rubro se encuentra la actividad física, por lo que se puede concluir que es de conocimiento de la población que el realizar actividad física genera beneficios para la salud. Bautista menciona que socialmente las consecuencias del padecimiento pueden modificar las relaciones del individuo con los demás de forma negativa (Bautista, 2005), ante esta situación, en el estudio se encontró que el cuidador incita a realizar actividades recreativas para fortalecer las relaciones sociales e interpersonales, siendo esto un beneficio para su salud. Por lo anterior, el apoyo familiar es fundamental para afrontar el duelo que conlleva la pérdida de su estilo de vida anterior e incorporarse a sus nuevos hábitos.

\section{CONCLUSIONES}

La Diabetes es un padecimiento crónico, que afecta de forma determinante la salud integral de los individuos que la padecen, y si se suma a ella el consumo de alcohol y/o tabaco éste padecimiento se vuelve aún más alarmante, ya que estas sustancias aceleran el deterioro orgánico del individuo, que se traduce en complicaciones propias de la enfermedad; por ello, es importante conocer el cuidado cultural que el familiar o persona cuidadora proporciona para el mantenimiento de la salud.

Con los resultados obtenidos se puede estipular que aunque la esperanza de vida ha aumentado, la calidad de vida de los individuos y su cuidador se ve en decremento potencia- 
lizando este deterioro, por lo cual, el cuidado de la Diabetes, el consumo de drogas lícitas, los malos hábitos alimenticios y las condiciones sociales y culturales de la población, se convierten en una área de oportunidad para el desarrollo de programas de enfermería enfocados a la promoción de la salud en el primer nivel de atención.

Es preciso denotar que las intervenciones de enfermería deberán de ser de manera integral, no solo para el enfermo sino también para todo el núcleo familiar, ya que representa un factor de adhesión positiva al tratamiento del diabético, sin dejar de lado la mejora en su estado emocional.

Parte importante de las acciones disciplinares radica en que enfermería contribuya a formular estrategias que permitan la sensibilización en los sujetos con Diabetes para adquirir nuevos conocimientos que beneficien la salud de manera integral y que permitan discernir entre las actividades que pueden afectar de manera negativa, no obstante, es importante mencionar que toda intervención debe ser siempre acorde con el nivel educativo.

Sabiendo que el alcohol y el tabaco son sustancias nocivas para la salud, es conveniente implementar intervenciones de enfermería enfocadas al conocimiento sobre los daños que el alcohol y el tabaco causan de manera aislada o en conjunto con la Diabetes en la salud del individuo, pues según Ngozi O. Nkongho, el cuidado compromete la participación humana, y con ella, el cuidador desarrolla habilidades para el cuidado de su familiar y de sí mismo.

\section{BIBLIOGRAFÍA:}

- Bautista, H. Influencia de los factores Estresores en el Manejo Inefectivo del Régimen Terapéutico del Paciente con DM tipo 2. [Manuscrito inédito].

- Díaz Álvarez, J.C., Rojas Martínez, M.V. (2009). Cuidando al cuidador: efectos de un programa educativo. Aquichan, 9 (1), 73-92.
- Fernández Ortega, M.A. (2007). El impacto de la enfermedad en la familia. Rev Fac Med UNAM, 6(47), 251254. Disponible en http://www.ejournal.unam.mx/rfm/ no47-6/RFM47606.pdf.

- Galván, M.C. (2011). Resiliencia y Motivación en el consumo de Alcohol y Tabaco en adolescentes de la telesecundaria (No. 28) de Rincón de Tamayo. Celaya, Gto. [Manuscrito inédito].

- Gómez Aguilar, P.S., Yam Sosa, A.V., Martín Pavó. M.J. (2010). Estilo de vida y Hemoglobina Glucosilada en la Diabetes Mellitus tipo 2. Rev Enferm Inst Mex Seguro Soc, 18 (2), 81-87.

- Guerrero-López, C.M., Muños-Hernández, J.A., Sáenz de Miera-Juárez, B., Reynales-Shigematsu, L.M. (2013). Consumo de tabaco, mortalidad y política fiscal en México. Rev salud pública de México, 55 (suplemento 2), S276-S281.

- Instituto Mexicano del Seguro Social (2009). Deportemania. Recuperado de http://www.deportemania.com.mx.

- Jacoby, E., Bull, F., Neiman, A. (2003). Cambios acelerados del Estilo de Vida obligan a fomentar la actividad física como prioridad en la región de las Américas. Revista: Panam. Salud Pública, 14 (4), 223-225.

- Organización de las Naciones Unidas. (2012). Asamblea General de las Naciones Unidas. Recuperado de http:// www.who.int.

- Polit D., Hungler B. (2000). Investigación científica en ciencias de la salud. México: McGraw-Hill Interamericana.

- Rondón Bernard, J.E. (2011). Variables psicosociales implicadas en el mantenimiento y control de la diabetes mellitus: Aspectos conceptuales, investigaciones y hallazgos. Revista electrónica de psicología Iztacala, 14 (2), 126-162.

- Salcedo Rocha, A.L., García de Alba García, J.E., \& Sevila, E. (2008). Dominio cultural del autocuidado en diabéticos tipo 2 con y sin control glucémico en México. Rev. Saúde Pública, 42 (2), 256-264.

- Secretaria de Salud. (2001). Programa de Acción: Adicciones Tabaquismo. México: Editorial SSA.

- Secretaria de Salud. (2008). Programa de Acción Específico (2007-2012) Diabetes Mellitus. México: Editorial SSA.

- Solís Torres, A., Alonso Castillo, M.M., \& López García, K.S. (2009). Prevalencia de consumo de alcohol en personas con diagnóstico de diabetes mellitus tipo 2. Revista Electrónica Salud Mental, Alcohol y Drogas, 5 (2), 1-13.

- Universia México. (09 de enero de 2014). Excesos en el consumo de alcohol: una realidad que afecta a la población mexicana. La Jornada. Recuperado de http://noticias.universia.net.mx/en-portada/ noticia/2014/01/08/1073512/excesos-consumo-alcoholrealidad-afecta-poblacion-mexicana.html

- Zavala, M.R., Vázquez Martínez, O., \& Whetsell, M.V. (2006). Bienestar espiritual y ansiedad en pacientes diabéticos. Aquichan, 6 (001), 8-21. 